\title{
A strongly truncated inner accretion disk in the Rapid Burster
}

\author{
J. van den Eijnden ${ }^{1,2}{ }^{\star}$, T. Bagnoli ${ }^{2,3}$, N. Degenaar ${ }^{1,2}$, A. M. Lohfink ${ }^{1}$, M. L. Parker ${ }^{1}$, \\ J. J. M in 't Zand ${ }^{3}$ and A. C. Fabian' \\ ${ }^{1}$ Institute of Astronomy, University of Cambridge, Madingley Road, Cambridge CB3 OHA, UK \\ ${ }^{2}$ Anton Pannekoek Institute for Astronomy, University of Amsterdam, Science Park 904, 1098 XH Amsterdam, The Netherlands \\ ${ }^{3}$ SRON Netherlands Institute for Space Research, Sorbonnelaan 2, 3584 CA Utrecht, the Netherlands
}

Accepted XXX. Received YYY; in original form ZZZ

\begin{abstract}
The neutron star (NS) low-mass X-ray binary (LMXB) the Rapid Burster (RB; MXB 1730-335) uniquely shows both Type-I and Type-II X-ray bursts. The origin of the latter is ill-understood but has been linked to magnetospheric gating of the accretion flow. We present a spectral analysis of simultaneous Swift, NuSTAR and XMM-Newton observations of the RB during its 2015 outburst. Although a broad Fe-K line has been observed before, the high quality of our observations allows us to model this line using relativistic reflection models for the first time. We find that the disk is strongly truncated at $41.8_{-5.3}^{+6.7}$ gravitational radii $(\sim 87 \mathrm{~km})$, which supports magnetospheric Type-II burst models and strongly disfavors models involving instabilities at the innermost stable circular orbit. Assuming that the RB magnetic field indeed truncates the disk, we find $B=(6.2 \pm 1.5) \times 10^{8} \mathrm{G}$, larger than typically inferred for NS LMXBs. In addition, we find a low inclination $\left(i=29 \pm 2^{\circ}\right)$. Finally, we comment on the origin of the Comptonized and thermal components in the RB spectrum.
\end{abstract}

Key words: accretion, accretion discs - X-rays: binaries - X-rays: individual: MXB 1730-335 - stars: neutron

\section{INTRODUCTION}

The Rapid Burster (MXB 1730-335, Lewin et al. 1976; hereafter RB) is a peculiar neutron star (NS) low-mass X-ray binary (LMXB) located at a distance of $7.9 \mathrm{kpc}$ in the globular cluster Liller-1 (Valenti et al. 2010). NS LMXBs often show X-ray bursts, either due to thermonuclear burning of accreted material on the NS surface (Type-I), or a sudden release of gravitational energy (Type-II). The RB is one of only two NSs showing Type-II X-ray bursts and the only source showing both types. It typically displays only Type-I bursts at high persistent luminosities, and both burst types at lower ones (Bagnoli et al. 2013). Various models for the poorly understood Type-II bursts have been proposed, including magnetospheric gating of the accretion flow (Spruit \& Taam 1993, see Bagnoli et al. 2015 for a recent overview of models), in which a strong NS magnetic field truncates the accretion disk outside the innermost stable circular orbit (ISCO; $6 R_{g}$ for a non-spinning NS, where $R_{g}=G M / c^{2}$ is the gravitational radius). Measuring the inner disk radius can thus provide a direct test of such magnetospheric models for the RB.

^ E-mail: a.j.vandeneijnden@uva.nl
Constraining the accretion geometry in LMXB is possible by modeling the reflection spectrum (Fabian et al. 1989): hard X-ray emission reflected off the accretion disk, which prominently contains a gravitationally and dynamically broadended Fe-K line at $\sim 6.5 \mathrm{keV}$. Using this approach, Degenaar et al. (2014) find an inner disk radius of $R_{\text {in }}=85.0 \pm 10.9 R_{g}$ in the Bursting Pulsar (GRO J174428, Kouveliotou et al. 1996; hereafter BP), the other source showing Type-II bursts. This truncation is much larger than typically observed in NS LMXBs $\left(6-15 R_{g}\right.$, see e.g. Cackett et al. 2010 for a sample study). Hence, the question arises whether a similar truncation is present in the RB. In this Letter, we present an analysis of new, simultaneous observations of the RB using Swift, NuSTAR and XMM-Newton, aimed at constraining the inner disk radius.

\section{OBSERVATIONS}

\subsection{Swift}

As RB outbursts are relatively predictable, a Swift (Gehrels et al. 2004) X-ray Telescope (XRT) monitoring campaign was carried out in Window Timing (WT) mode to detect 
the start of the outburst. An outburst was detected on 2015 October 3 and triggered, simultaneous $N u S T A R$ and $X M M$ Newton observations were performed on October 6. A single $\sim 500 \mathrm{~s}$ Swift observation (obsID 00031360129) coincided with the NuSTAR and XMM-Newton observations. For this observation, we use XSELECT v2.4d to extract an XRT spectrum from a 70.8 arcsec radius aperture. We create an arf using XRTMKARF, take the rmf (v15) from the CALDB, and rebin the spectrum to ensure a minimum of 20 counts per bin using GRPPHA. As Swift-WT observations of bright, absorbed sources tend to show residuals below $\sim 1 \mathrm{keV}^{1}$, we fit the Swift spectrum only in the $1-10 \mathrm{keV}$ range.

\section{$2.2 \quad N u S T A R$}

NuSTAR (Harrison et al. 2013) observed the RB between 2015 October 6 12:11:08 and October 7 15:11:08 (obsID 90101009002 ), amounting to $\sim 46 \mathrm{ks}$ on-target exposure time for both Focal Plane Modules (FMP) A and B. This full exposure consists of $\sim 40 \mathrm{ks}$ non-burst and $\sim 6 \mathrm{ks}$ burst exposure (see Section 3 for the determination of burst intervals). We apply the standard routines NUPIPELINE and NUPRODUCTS to extract source and background spectra for the non-burst and burst intervals seperately. We extract source spectra from a 120 arcsec radius circular aperture. As the source dominates its chip, we extract background spectra from a same-sized region on the opposite chip. However, we find no significant differences with background spectra extracted from either the two remaining chips or a smaller background region on the source chip. The source spectrum dominates above the background up to $\sim 30 \mathrm{keV}$, so we fit the NUSTAR spectra in the range $3-30 \mathrm{keV}$.

\section{$2.3 \quad X M M-N e w t o n$}

XMM-Newton (Jansen et al. 2001) observed the RB between 2015 October 6 19:23:44 and October 7 06:12:20 (obsID 0770580601) with the EPIC-pn in timing mode and MOS1/2 turned off, resulting in $\sim 18 \mathrm{ks}$ on-target exposure (of which $\sim 3 \mathrm{ks}$ burst exposure). We process the Observation Data Files using XMM-Newton SAS v15. For the RGS detector, we apply RGSPROC to extract event lists for non-burst and burst intervals seperately. After assuring no background flaring is present, we combine spectra of the same order from the two RGS detectors using RGSCOMBINE and rebin to a minimum of 20 counts per bin. The extracted spectra show discrepancies between the two orders, and between the RGS and XRT spectra, below 1 and above $2 \mathrm{keV}$. Hence, we only consider the range 1-2 keV for the RGS spectra. In this work, we do not search for narrow features in the RGS spectra.

The EPIC-pn spectrum (extracted using EPPROC and EPCHAIN with quality flag $=0$, pattern $\leq 4$ ) shows crosscalibration issues with the Swift and NuSTAR spectra: the continuum slope above $\sim 5 \mathrm{keV}$ differs significantly between the spectra. Similar issues in timing mode observations of black-hole binaries in the hard state have been reported recently (see e.g. Ingram et al. 2016). Given the consistency between the Swift and NuSTAR spectra, we decide to exclude the EPIC-pn spectra.

1 See http://www.swift.ac.uk/analysis/xrt/digest_cal.php\#abs.

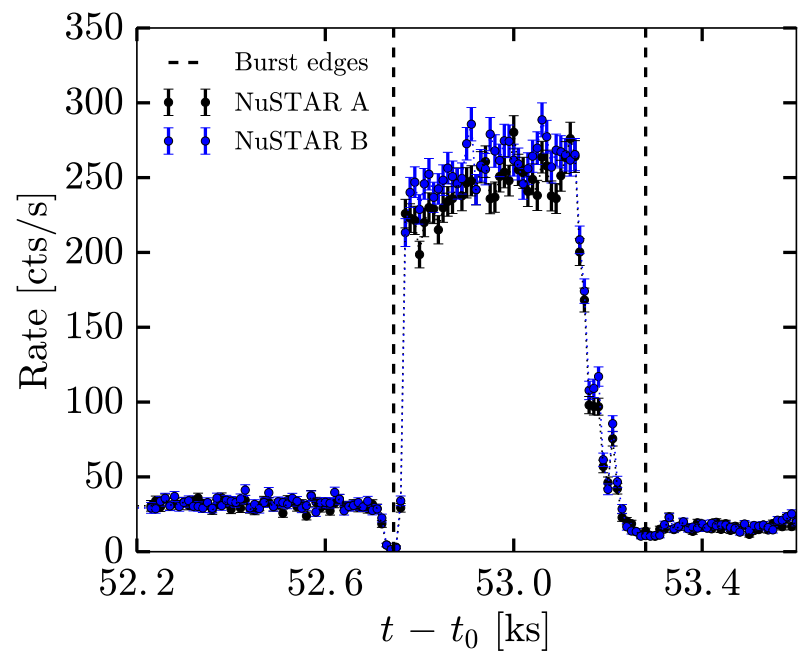

Figure 1. Representative example of a Type-II burst in the $N u S$ $T A R$ observation. The characteric dips, visible before and after the burst, form the border between the non-burst and burst GTIs. $t_{0}$ is the start time of the observation.

\section{SPECTRAL FITTING}

The NuSTAR and XMM-Newton light curves contain no Type-I bursts and in total 56 Type-II bursts, an example of which is shown in Fig. 1. Measuring the time of the minima in the characteristic dips before and after each burst, we manually define the non-burst and burst intervals used in the extraction of the spectra. Per instrument, we extract a single burst spectrum combining all Type-II bursts. This burst spectrum contains $\sim 47 \%$ (FMPA,FMPB) and $\sim 39 \%$ (RGS) of the total counts in the observation. The Swift observation coincides fully with a non-burst interval. We initially focus on the non-burst spectrum, and discuss on the burst spectrum at the end of Section 3.2.

We use XSPEC v12.9.0 (Arnaud 1996) for the spectral fitting and assume solar abundances from Wilms et al. (2000) and cross-sections from Verner et al. (1996). We model interstellar absorption using TBABS and include a free constant between all spectra fixed to 1 for the FMPA spectrum). All quoted uncertainties are at $1 \sigma$.

\subsection{Phenomenological modelling}

Falanga et al. (2004) fit an Integral spectrum (3-100 keV) of the RB with a model consisting of a powerlaw, a blackbody and a Gaussian Fe-K line. We adopt a similar approach, but replace the powerlaw with the physically-motivated Comptonization model COMPTT (Titarchuk 1994). This model yields a reasonable fit with $\chi_{\nu}^{2}=1.32(3265 / 2477)$, and the inclusion of the blackbody component is required at high significance $\left(\Delta \chi^{2} / \Delta\right.$ d.o.f $\left.=277 / 2\right)$. For the Gaussian component, we measure $E_{\mathrm{G}}=6.50 \pm 0.02 \mathrm{keV}$ and $\sigma_{\mathrm{G}}=0.86 \pm 0.03$ $\mathrm{keV}$, consistent with Falanga et al. (2004). However, this phenomenological modelling does not provide us with a statistically satisfactory fit, and a Gaussian does not adequately describe the feature around $\sim 6.5 \mathrm{keV}$. Hence, we turn to relativistic reflection modelling of the non-burst spectrum. 


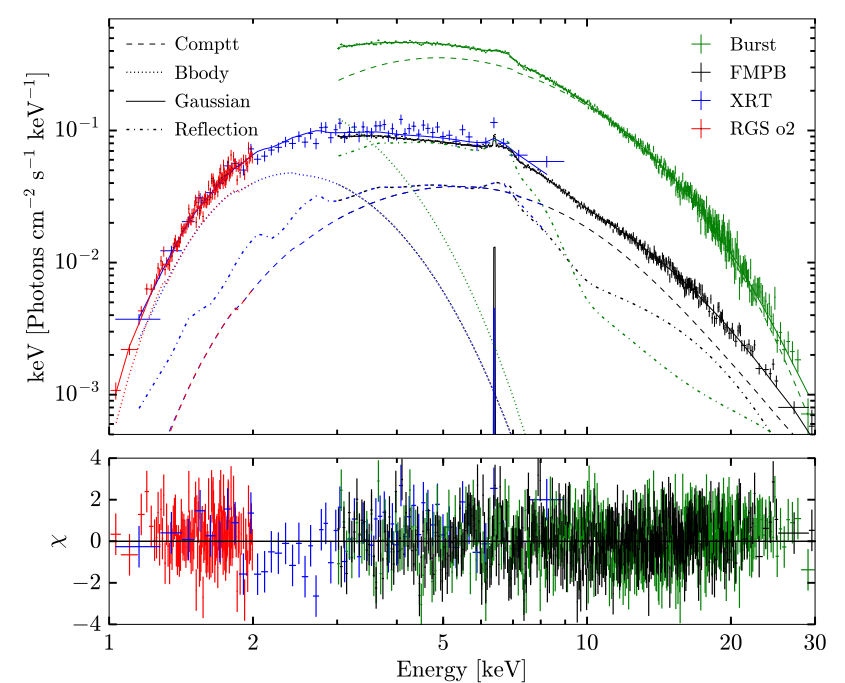

Figure 2. The NuSTAR (FMPB), XMM-Newton RGS (order 2) and Swift unfolded spectra with the best fitting TBABS (COMPTT + BBODYRAD + RELCONV*REFLIONX + GAUSS) model. For clarity, we show only a single spectrum per telescope. For comparison, we also show the unfolded FMPA burst spectrum in green. The spectra have been rebinned for visual purposes in XSPEC using SETPLOT REBIN. Note that small deviations appear visible in the Swift-spectrum between 2 and $3 \mathrm{keV}$.

\subsection{Relativistic reflection modelling}

For our reflection fits, we replace the Gaussian line with the model REFLIONX (Ross \& Fabian 2005). We apply an adapted version of this model, which was calculated with a COMPTT illuminating spectrum instead of a powerlaw ${ }^{2}$, as COMPTT is dominant in the phenomenological fit at all energies. To include relativistic effects, we convolve REFLIONX with RELCONV (Dauser et al. 2010). We link the input soft photon temperature $T_{0}$, electron temperature $k T_{e}$ and optical depth $\tau$ between COMPTT and REFLIONX. We fix the dimensionless spin parameter $a=0.0$, as for NSs this value ranges from 0.0 to 0.3 and has little effect on the surrounding metric (see e.g. Miller et al. 1998). Indeed, setting $a=0.3$ does not yield significant changes in either the model parameters or the quality of the fit. Furthermore, we assume an unbroken emissivity profile with a fixed slope of $q=3$, as the slope is not contrained by the data. This value is consistent with both theoretical expectations (Wilkins \& Fabian 2012) and results from a sample study in NS LMXBs by Cackett et al. (2010). The disk ionisation, parametrised as $\xi \equiv 4 \pi F / n$, where $F$ is the illuminating flux and $n$ the hydrogen number density, is left variable. In addition, we leave the inclination $i$, inner disk radius $R_{\mathrm{in}}$, and iron abundance $A_{\mathrm{Fe}}$ free to vary.

We attempt two possibilities for a soft component: DISKBB and BBODYRAD (the complete models are hereafter referred to as the disk- and BB-model, respectively). Furthermore, inspection of the residuals around the Fe-K line

\footnotetext{
2 http://www-xray.ast.cam.ac.uk/ mlparker/reflionx_models/
} reflionx_comptt_hightau.mod
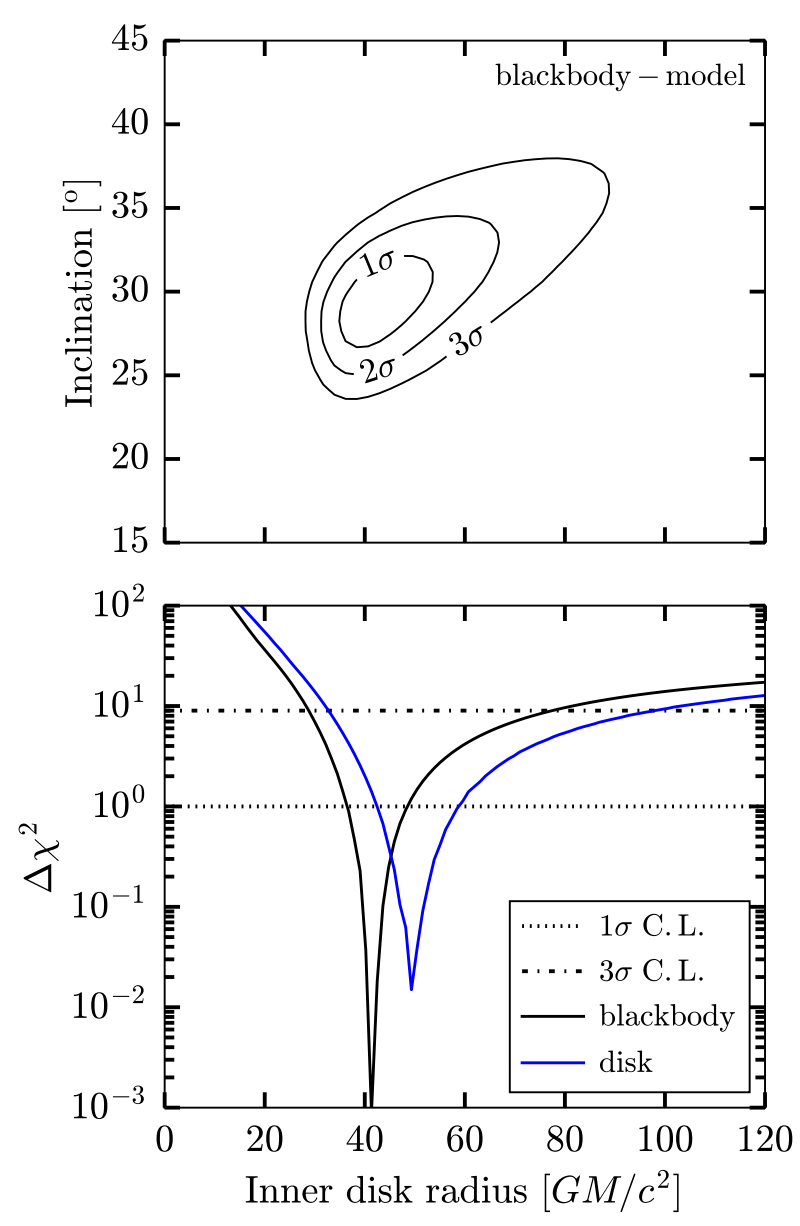

Figure 3. Top: $2 \mathrm{D}$ confidence contour for the inclination and inner disk radius or the BB-model. Bottom: confidence plot of the inner disk radius for the blackbody and disk-models.

suggests the presence of an additional narrow emission line around $6.4 \mathrm{keV}$. Hence, we also include a narrow Gaussian $\left(\sigma=10^{-3}\right)$ fixed at this energy of $6.4 \mathrm{keV}$.

The disk-model yields a good fit, with $\chi_{\nu}^{2}=1.13$ $(2789 / 2472)$ for a temperature $k T_{\text {disk }}=0.73_{-0.01}^{+0.02} \mathrm{keV}$. The BB-model results in the best fit, with $\chi_{\nu}^{2}=1.10$ $(2727.8 / 2472)$ for a temperature $k T_{\mathrm{bb}}=0.55 \pm 0.01 \mathrm{keV}$. All other parameters are listed in Table 1. Most interestingly, the reflection component implies a large disk truncation in both models: $R_{\text {in }}=41.8_{-5.3}^{+6.7} R_{g}$ for the BB-model and $R_{\text {in }}=49.5_{-7.0}^{+9.2} R_{g}$ for the disk-model, both significantly larger than commonly observed in NS LMXBs (e.g. Cackett et al. 2010). Both models also yield a consistent, low inclination estimate of $\sim 30^{\circ}$ and an intermediate disk ionisation of $\xi \sim 470$. The latter is consistent with the typical range observed in both black hole and NS LMXBs $(\log (\xi) \sim 2-3)$. The BB-model yields an unabsorped flux between $1-30 \mathrm{keV}$ of $1.47 \times 10^{-9} \mathrm{erg} \mathrm{cm}^{-2} \mathrm{~s}^{-1}$, corresponding to a luminosity of $1.23 \times 10^{37} \mathrm{erg} \mathrm{s}^{-1}$ (at a distance of $7.9 \mathrm{kpc}$ ) and an Eddington ratio of $\sim 3.2 \%$ assuming the emperical Eddington luminosity determined by Kuulkers et al. (2003).

Fig. 2 shows the spectra and the best-fitting relativistic 
Table 1. Model parameters for the TBABS(COMPTT + GAUSS+soft component+RELCONV*REFLIONX)-models. All quoted uncertainties are at $1 \sigma$. We fix $q=3$ and $a=0.0$.

\begin{tabular}{llcc}
\hline \hline Component & Par. [Unit] & BB-model & Disk-model \\
\hline TBABS & $N_{\mathrm{H}}\left[10^{22} \mathrm{~cm}^{-2}\right]$ & $3.17 \pm 0.03$ & $3.64 \pm 0.03$ \\
COMPTT & $T_{0}[\mathrm{keV}]$ & $1.54 \pm 0.01$ & $1.56 \pm 0.01$ \\
& $k T_{e}[\mathrm{keV}]$ & $7.19 \pm 0.08$ & $7.12 \pm 0.08$ \\
& $\tau$ & $1.01_{-0.11}^{+0.30}$ & $1.00_{-0.11}^{+0.20}$ \\
& Norm $\left[10^{-2}\right]$ & $1.07 \pm 0.03$ & $1.10 \pm 0.03$ \\
GAUSS & Norm $\left[10^{-4}\right]$ & $1.66 \pm 0.35$ & $1.83 \pm 0.34$ \\
DISKBB & $k T_{\text {disk }}[\mathrm{keV}]$ & - & $0.73_{-0.01}^{+0.02}$ \\
& Norm & - & $138 \pm 15$ \\
BBODYRAD & $k T_{\mathrm{BB}}[\mathrm{keV}]$ & $0.55 \pm 0.01$ & - \\
& Norm & $450 \pm 40$ & - \\
RELCONV & $i\left[{ }^{\circ}\right]$ & $29 \pm 2$ & $32 \pm 2$ \\
& $R_{\text {in }}\left[R_{g}\right]$ & $41.8_{-5.3}^{+6.7}$ & $49.5_{-7.0}^{+9.2}$ \\
REFLIONX & $\xi$ & $470_{-14}^{+61}$ & $460 \pm 10$ \\
& $A_{\mathrm{Fe}}$ & $0.71_{-0.06}^{+0.08}$ & $0.77 \pm 0.06$ \\
& Norm & $10.8_{-0.9}^{+0.4}$ & $10.4_{-0.5}^{+0.3}$ \\
\hline
\end{tabular}

reflection + blackbody model. Fig. 3 shows the confidence contours for the inner disk radius and the inclination. Both parameters are clearly well constrained by the data, and the inner disk radius is inconsistent with the ISCO (i.e. $6 R_{g}$ ) at $\gtrsim 14.1 \sigma\left(\Delta \chi^{2} \gtrsim 200\right)$ for either model.

The emission line at $6.4 \mathrm{keV}$ is significant at $\sim 5 \sigma$ for both models, given the uncertainty in the normalization. This line, consistent with neutral iron, is also seen in the BP (Degenaar et al. 2014), although it is more generally observed in high-mass X-ray binaries (Torrejón et al. 2010). Letting its energy vary does not provide a significant improvement of the fit (f-test probability $p>0.01$ for both models). The residuals in Fig. 2 also suggest the presence of an absorption line around $6.9 \mathrm{keV}$. However, the addition of a Gaussian absorption line is not statistically significant.

Fitting the BB-model to the RGS and NuSTAR burst spectra (see Fig. 2) yields a poorly constrained inclination and $R_{\text {in }}$. Thus, we instead fit the burst and non-burst spectra simultaneously, tying the column density and inclination. This results in a good fit $\left(\chi_{\nu}^{2}=1.11(4744.1 / 4272)\right)$ as now the non-burst data constrains the inclination at $i=22 \pm 1^{\circ}$. We measure $R_{\text {in }}=40.1_{-2.8}^{+3.3} \quad R_{g}$, consistent with the nonburst value. $R_{\text {in }}$ is inconsistent with the ISCO at $\gtrsim 8 \sigma$. The burst spectrum is significantly softer, yielding a lower electron temperature of $k T_{e}=2.42 \pm 0.03 \mathrm{keV}$. Finally, the Comptonized component is much stronger relative to the reflection spectrum during burst intervals.

\section{DISCUSSION}

We present a spectral analysis of simultaneous $N u S T A R$, $X M M$-Newton and Swift observations of the RB, aimed at contraining its accretion geometry and the origin of its peculiar Type-II burst behaviour. The non-burst spectrum is well described by a combination of the Comptonization model COMPTT, relativistic reflection of this Comptonized emission (RELCONV*REFLIONX), a soft (disk)blackbody and a narrow emission line at $6.4 \mathrm{keV}$. From the reflection spectrum, we measure a large inner disk truncation radius $(\sim 40-50$
$\left.R_{g}\right)$ and a low inclination $\left(\sim 30^{\circ}\right)$. Here, we will discuss the nature of the disk truncation, the implications for Type-II burst models, and the origin of the spectral components.

\subsection{The nature of the truncated disk}

NS LMXBs show a wide range of inferred inner disk radii, which can roughly be divided into three categories: most sources show small inner disk radii of 6-15 $R_{g}$ (12 LMXBs, see Cackett et al. 2010; Degenaar et al. 2015; Di Salvo et al. 2015; Ludlam et al. 2016; Sleator et al. 2016). Secondly, five NS LMXBs, mostly (intermittent) X-ray pulsars, show a slightly larger inner radius of roughly $15-30 R_{g}$ (Iaria et al. 2016; Miller et al. 2011; Papitto et al. 2013; Pintore et al. 2016; King et al. 2016). Finally, two sources show significantly larger truncation radii: Degenaar et al. (2016) infer $R_{\text {in }} \gtrsim 100 R_{g}$ for IGR J17062-6143, an LMXB persistently accreting a low rates $\left(L_{X} \approx 4 \times 10^{35} \mathrm{erg} \mathrm{s}^{-1}\right)$. Although various possible explanations exist for a truncated disk at such low $L_{X}$, truncation by the magnetosphere would imply a large NS magnetic field $\left(\gtrsim 4 \times 10^{8} \mathrm{G}\right)$. Furthermore, Degenaar et al. (2014) measure $R_{\text {in }}=85.0 \pm 10.9 R_{g}$ in the BP. Our result places the RB in this third category of large disk truncations. As only the RB and the BP show Type-II bursts, the presence of a large disk truncation in both forms an interesting constraint of Type-II burst models.

Models for Type-II bursts can be divided into different general categories (see Bagnoli et al. 2015, for a review): instabilities in the accretion flow, general-relativistic instabilities close to the ISCO, and interactions between the accretion disk and the NS magnetic field. As only two LMXBs show Type-II bursts, it is difficult to distinguish between these options. However, the first type of model is unable to account for the uniqueness of the RB and the BP: such instabilities should be observed more generally among NS LMXBs. The second category is strongly disfavored by our inner disk radius measurement, as it requires the disk to extend up to the ISCO.

The most prominent model of accretion instabilities driven by a disk-magnetic field interaction is a so-called trapped disk (Spruit \& Taam 1993; D'Angelo \& Spruit 2010). At the magnetospheric radius $R_{m}$, the disk is truncated by the magnetic field (see e.g. Pringle \& Rees 1972). If the NS spin frequency exceeds the Keplerian rotation frequency of the disk at this radius $R_{m}$, the magnetic field prevents accretion, confining infalling material in the disk (Sunyaev \& Shakura 1977; Spruit \& Taam 1993), or, alternatively, resulting in outflows (Illarionov \& Sunyaev 1975). As matter piles up, the magnetospheric radius moves inwards, until the Keplerian frequency exceeds the NS spin, matter swiftly accretes and $R_{m}$ moves out again. This trapped-disk model is consistent with our measurement of a large truncation radius in the $\mathrm{RB}$, and the similar result for the BP by Degenaar et al. (2014). Additionally, in this scenario a change in $R_{\text {in }}$ might be expected during bursts. However, the size of such a change in inner radius is unknown; given the relatively large uncertainties on $R_{\text {in }}\left(5-10 R_{g}\right)$, we might simply be unable to detect such changes significantly in our observations.

Assuming that the disk is truncated by the magnetic field, we can estimate the magnetic field of the RB using Equation 1 in Cackett et al. (2009). We estimate a bolometric flux of $F_{\text {bol }}=(2.25 \pm 0.2) \times 10^{-9} \mathrm{erg} \mathrm{cm}^{-2} \mathrm{~s}^{-1}$ by extrapo- 
lating the best fit over the $0.1-100 \mathrm{keV}$ range. Using geometrical and efficiency parameters from Cackett et al. (2009), we find $B=(6.2 \pm 1.5) \times 10^{8}\left(M / 1.4 M_{\odot}\right)^{2}(R / 10 \mathrm{~km})^{-3} \mathrm{G}$. This estimate is higher than generally observed for NS LMXBs (Mukherjee et al. 2015). A similarly high magnetic field as in the RB is present in the $11-\mathrm{Hz}$ pulsar IGR J17480-2446 in Terzan 5 (Miller et al. 2011). This strengthens the proposed link between these two sources (Bagnoli et al. 2013) and indicates that the Rapid Burster, like IGR J17480-2446, could be young, mildly recycled LMXB (Patruno et al. 2012). However, a spin measurement for the RB remains necessary to confirm this scenario.

\subsection{Completing the geometrical picture}

Falanga et al. (2004) fit the continuum in a 3-100 keV Integral spectrum with a combination of a powerlaw and a blackbody. The blackbody parameters $\left(k T_{\mathrm{BB}} \sim 2.2 \mathrm{keV}\right.$, $\left.R_{\mathrm{BB}} \sim 1.4 \mathrm{~km}\right)$ suggest that this continuum might originate from a hotspot on the NS surface, as expected if the magnetic field truncates the disk, or a boundery layer. Instead, we fit the continuum above $3 \mathrm{keV}$ using a COMPTTcomponent, which might thus arise from such a hotspot or boundary layer (instead of e.g. a corona). In the trapped-disk model, such an origin could also explain the large increase in the COMPTT-flux during bursts.

As we also have high-quality data below $3 \mathrm{keV}$, we detect an additional soft blackbody component unseen by $I n$ tegral. Its temperature is consistent with the expectation for the NS surface at the inferred accretion rate (Zampieri et al. $1995)$, although the implied radius of $16.7 \mathrm{~km}$ is larger than expected for NSs $(\sim 10 \mathrm{~km})$. However, Zampieri et al. (1995) also show that the surface spectrum might slightly deviate from a perfect blackbody. These deviations in the surface spectrum might thus explain why the BBODYRAD model infers a larger radius than expected. If the soft component indeed corresponds to the NS surface, it completes a selfconsistent geometrical description of the RB spectrum with a large truncation radius and a hotspot on the NS surface.

\section{ACKNOWLEDGEMENTS}

We thank the referee for comments on this Letter. JvdE and ND are supported by a Vidi grant from the Netherlands Organization for Scientific Research (NWO) awarded to ND. ND also acknowledges support via a Marie Curie fellowship (FP-PEOPLE-2013-IEF-627148) from the European Commission. ACF, AL and MP are supported by Advanced Grant Feedback 340442 from the European Research Counsil (ERC). TB acknowledges support from NewCompStar (COST Action MP1304). JvdE and TB acknowledge the hospitality of the Institute of Astronomy in Cambridge, where this research was carried out. We are grateful to Fiona Harrison, Norbert Schartel, Neil Gehrels and the observation planners for making these Director's Discretionary Time observations possible.

\section{REFERENCES}

Arnaud K. A., 1996, in Jacoby G. H., Barnes J., eds, Astronomical Society of the Pacific Conference Series Vol. 101, Astronomical Data Analysis Software and Systems V. p. 17

Bagnoli T., in't Zand J. J. M., Galloway D. K., Watts A. L., 2013, MNRAS, 431, 1947

Bagnoli T., in't Zand J. J. M., D'Angelo C. R., Galloway D. K., 2015, MNRAS, 449, 268

Cackett E. M., Altamirano D., Patruno A., Miller J. M., Reynolds M., Linares M., Wijnands R., 2009, ApJ, 694, L21

Cackett E. M., et al., 2010, ApJ, 720, 205

D'Angelo C. R., Spruit H. C., 2010, MNRAS, 406, 1208

Dauser T., Wilms J., Reynolds C. S., Brenneman L. W., 2010, MNRAS, 409, 1534

Degenaar N., Miller J. M., Harrison F. A., Kennea J. A., Kouveliotou C., Younes G., 2014, ApJ, 796, L9

Degenaar N., Miller J. M., Chakrabarty D., Harrison F. A., Kara E., Fabian A. C., 2015, MNRAS, 451, L85

Degenaar N., Pinto C., Miller J. M., Wijnands R., Altamirano D., Paerels F., Fabian A. C., Chakrabarty D., 2016, MNRAS,

Di Salvo T., et al., 2015, MNRAS, 449, 2794

Fabian A. C., Rees M. J., Stella L., White N. E., 1989, MNRAS, 238,729

Falanga M., Farinelli R., Goldoni P., Frontera F., Goldwurm A., Stella L., 2004, A\&A, 426, 979

Gehrels N., et al., 2004, ApJ, 611, 1005

Harrison F. A., et al., 2013, ApJ, 770, 103

Iaria R., et al., 2016, preprint, (arXiv:1609.06487)

Illarionov A. F., Sunyaev R. A., 1975, A\&A, 39, 185

Ingram A., Van der Klis M., Middleton M., Altamirano D., Uttley P., 2016, MNRAS

Jansen F., et al., 2001, A\&A, 365, L1

King A. L., et al., 2016, ApJ, 819, L29

Kouveliotou C., van Paradijs J., Fishman G. J., Briggs M. S., Kommers J., Harmon B. A., Meegan C. A., Lewin W. H. G., 1996, Nature, 379, 799

Kuulkers E., den Hartog P. R., in't Zand J. J. M., Verbunt F. W. M., Harris W. E., Cocchi M., 2003, A\&A, 399, 663

Lewin W. H. G., et al., 1976, ApJ, 207, L95

Ludlam R. M., et al., 2016, ApJ, 824, 37

Miller M. C., Lamb F. K., Cook G. B., 1998, ApJ, 509, 793

Miller J. M., Maitra D., Cackett E. M., Bhattacharyya S., Strohmayer T. E., 2011, ApJ, 731, L7

Mukherjee D., Bult P., van der Klis M., Bhattacharya D., 2015, MNRAS, 452, 3994

Papitto A., et al., 2013, MNRAS, 429, 3411

Patruno A., Alpar M. A., van der Klis M., van den Heuvel E. P. J., 2012, ApJ, 752, 33

Pintore F., et al., 2016, MNRAS, 457, 2988

Pringle J. E., Rees M. J., 1972, A\&A, 21, 1

Ross R. R., Fabian A. C., 2005, MNRAS, 358, 211

Sleator C. C., et al., 2016, ApJ, 827, 134

Spruit H. C., Taam R. E., 1993, ApJ, 402, 593

Sunyaev R. A., Shakura N. I., 1977, Soviet Astronomy Letters, 3, 138

Titarchuk L., 1994, ApJ, 434, 570

Torrejón J. M., Schulz N. S., Nowak M. A., Kallman T. R., 2010, ApJ, 715, 947

Valenti E., Ferraro F. R., Origlia L., 2010, MNRAS, 402, 1729

Verner D. A., Ferland G. J., Korista K. T., Yakovlev D. G., 1996, ApJ, 465, 487

Wilkins D. R., Fabian A. C., 2012, MNRAS, 424, 1284

Wilms J., Allen A., McCray R., 2000, ApJ, 542, 914

Zampieri L., Turolla R., Zane S., Treves A., 1995, ApJ, 439, 849 
L6 Van den Eijnden et al.

This paper has been typeset from a $\mathrm{T}_{\mathrm{E}} \mathrm{X} / \mathrm{LAT}_{\mathrm{E}} \mathrm{X}$ file prepared by the author. 